\title{
Field Performance of BAU-Biofungicaide for Management of Narrow Brown Leaf Spot and Bacterial Leaf Blight Disease of Rice CV. BRRI Dhan29
}

\section{Hyat Mahmud ${ }^{1 *}$, Ismail Hossain², Muhammad Emdadul Haque ${ }^{3}$ and Kamal Hasan AKM ${ }^{4}$}

${ }^{1}$ Chief Instructor, Agriculture Training Institute, Department of Agricultural Extension, Ishwardi, Pabna, Bangladesh

${ }^{2}$ Professor, College of Agricultural sciences, International University of Business Agriculture and Technology (IUBAT), Dhaka, Bangladesh

${ }^{3}$ Deputy Director, Horticulture Centre, Department of Agricultural Extension, Mouchak, Gazipur, Bangladesh

${ }^{4} \mathrm{PhD}$ fellow, Department of Plant Pathology, Bangladesh Agricultural University (BAU), Mymensingh, Bangladesh

*Corresponding author: Hyat Mahmud, Chief Instructor, Agriculture Training Institute, Department of Agricultural Extension, Ishwardi, Pabna, Bangladesh, Tel: 01727182615, Email: mhyat81@gmail.com

\section{Abstract}

Potent $(0.1 \%)$ and BAU-Biofungicide (2\%) revealed the significant reduction both in disease severity and incidence of narrow brown leaf spot disease, and the lowest disease severity and incidence of bacterial leaf blight disease were $3.00 \%$ and $5.83 \%$, respectively in BRRI dhan29 in 2012 year. BAU-Biofungicide (Trichoderma based preparation), extract of garlic (Allium sativum) and neem (Azadirachta indica), Bavistin DF (Carbandazim) and Potent 250 EC (Propiconazole) were evaluated in controlling narrow brown leaf spot and bacterial leaf blight disease (Xanthomonas oryzae pv. Oryzae) under field condition for producing seed quality and controlling seed borne pathogen of rice cv BRRI dhan29 during 2012 and 2013 years. Maximum normal seedling (87.67\%) and 37.49\% higher increase in vigour index on seed quality test of harvested seeds were observed with BAU-Biofungicide (3\%) over control. BAU-Biofungicide ( 2 and 3\%) and Potent 250 EC (0.1\%) were found to be effective in controlling seed borne pathogens. BAU-Biofungicide has also been attributed as profound contribution on quality seed production of harvested seed of BAU-Biofungicide sprayed plot.

Keywords: BAU-Biofungicide; Cercospora oryzae Miyake; Potent; Seed borne pathogen; Seed quality; Xanthomonas oryzae pv. Oryzae

\section{Introduction}

Rice (Oryza sativa L.) is the most important staple food crop in Bangladesh. Its cultivation is approximately 11.91 million hectares and total rice production is 56.42 million tons [1]. Average yield of rice is $4.74 \mathrm{t} / \mathrm{ha}$ which is very lower in compared to neighboring countries, whereas Japan produce $6.62 \mathrm{t} / \mathrm{ha}$ [1]. Many factors of biotic and abiotic stresses are responsible for the low yield of rice in our country. However, the rice disease is considered to be the major constraint for sustaining rice productivity. The rice production environments in the tropics having many rice pathogens cause varying degrees of devastating damage. Of these, the narrow brown leaf spot is distributed in the worldwide as foliar disease and prevalent in global rice grown areas [2,3], and its yield loss has also been reported in Bangladesh [4]. Narrow brown leaf spot disease is caused by Cercospora oryzae Miyake and its infection proceeds 
on leaves, sheaths and panicles [5]. The pathogen is also airborne and endures in crop residue and on seed. In respect to susceptible cultivar, significant economic yield losses were reported with worm weather [6]. Bacterial leaf blight (BLB) is the most important vascular disease which is difficult to control, and uses of higher nitrogen rate produced higher grain yield, while yield was greatly reduced due to BLB [7]. Bacterial leaf blight (Xanthomonas oryzae pv. Oryzae) disease in rice causes epidemic losses in all rice growing areas of the world and even destructive in Asia [8,9]. Disease injury causes significant reductions in filled grains and yield loss ranges from $31-44 \%$ [10].

Seeds play a vital role to produce quality and healthy seeds which has the significant effect on yield of rice. About $40 \%$ of the rice seeds are obtained from farmers' own harvest and $60 \%$ come from the Bangladesh Agricultural Development Corporation (BADC), Non governmental organizations (NGOs) and private seed companies in Bangladesh. Meanwhile, farmers - saved rice seeds are also of magnificence in carrying seed-borne pathogens [11]. The certified seeds are recognized as good quality seeds in the country, but the quality of these seeds are judged only by moisture, purity and germination, where the health of seeds is not considered. Quality good seed consists of major characteristics such as high yielding potentiality, viability and free from infection by pathogens [12]. Seed borne infection of rice seed by Bipolaris oryzae (brown spot), Fusarium moniliforme (bakanae) and Alternaria padwiickii (stack burn), F. oxysporum, Curvularia lunata, species of Nigrospora, Aspergillus flavus and Sarocladium oryzae (sheath rot) greatly affects in seed quality [13]. These organisms cause grain discoloration and reduce market value. Poor quality rice seeds reduce the productivity of modern cultivars in attaining its genetic potential [14].

Application of fungicides for the control of plant diseases is the most effective management option. Moreover, indiscriminate use of chemicals breaks down the natural ecological balance by killing the beneficial and/or antagonistic soil microbes. However, exploring of judicious, less expensive, less hazardous and eco-friendly management practices are essential tool to combat the problem [15]. Bioagents have also been attempted in minimizing the disease to reduce crop losses [16,17]. BAU-Biofungicide (Trichoderma based preparation) results in significant higher germination and plant resistance, less disease incidence and higher yield of rice [18]. Trichoderma spp. produces hydrolytic enzymes (chitinases, glucanases, and proteases) to breach the fungal cell wall as successful invasion in the fungal hosts $[19,20]$ and releases antimicrobial metabolites act as hyperparasitism [21]. Plant extract as garlic has its highly significant antifungal function and antimicrobial properties [22,23] and extract of neem also shows antifungal effects $[18,24,25]$. The present study has been designed as eco-friendly management of the narrow brown leaf spot and bacterial leaf blight diseases of rice for improving seed quality and controlling seed borne pathogens.

\section{Materials and Method}

\section{Use of BAU-Biofungicide and Fungicide}

BAU-Biofungicide (Trichoderma based preparation) was used at $2 \%$ and $3 \%$. BAU-Biofungicide is a Trichoderma based preparation [26]. Bavistin DF (Carbendazim) and Potent 250 EC (Propiconazole) were also used at $0.1 \%$ and $0.05 \%$ concentration.

\section{Preparation of Plant Extracts}

Healthy leaves of neem and garlic cloves were collected, and washed thoroughly under running tap water followed by sterile distilled water (SDW). The extracts were prepared by homogenizing plant parts using a blender and prepared at $1 \%$ and $2 \%$ concentration by dilution with water and kept in conical flasks separately before use.

\section{Field Experiments}

The experiments were conducted with rice cv. BRRI dhan29 during two Boro seasons in two successive years 2012 and 2013. The experiments were carried out in the field Laboratory of the Department of Agronomy, Bangladesh Agricultural University (BAU), Mymensingh. The experiment was led by using Randomized Complete Block Design (RCBD) having three replications. The field was fertilized as per recommendation of Bangladesh Rice Research Institute, Gazipur [27]. The individual plot size was $10 \mathrm{~m}^{2}$. Block to block and plot to plot distances were $2.0 \mathrm{~m}$ and $1.5 \mathrm{~m}$, respectively. Thirty five day old seedlings were uprooted from seed bed and three seedlings per hill were transplanted on January 21 in two successive years 2012 and 2013. Hill to hill and row to row distances were $15 \mathrm{~cm}$ and $20 \mathrm{~cm}$, respectively. The spray schedule was started just after commencement of disease symptoms and three sprays were followed at 15 days interval. Symptoms have been assessed at 80, 95 and 110 days after transplantation. Disease severity and incidence of each plot was assessed following the procedure of Standard Evaluation System for Rice [28].

\section{Tray Method}

The experiment was conducted in the nethouse of the Seed Pathology Centre, (BAU), Mymensingh. Sand was collected from Brahmaputra River, Mymensingh. The collected sand was sterilized with formalin (40\%) at the rate of $5 \mathrm{ml}$ formalin Diluted with $20 \mathrm{ml}$ of water for $4 \mathrm{~kg}$ sand [29]. The formalin treated soil was covered with polythene 
sheet for $48 \mathrm{~h}$ and then exposed for $48 \mathrm{~h}$ for aeration before setting experiment. The plastic trays $\left(12^{\prime \prime} \times 8^{\prime \prime}\right)$ were filled with the sand. The experiment was carried out in Complete Randomized Design with three replications. Three hundred harvested seeds of each treatment including control were sown in plastic trays (100 seeds/tray) maintaining equal distances among the seeds. Plants were watered as when necessary for maintaining proper moisture. Randomly selected 10 seedlings were uprooted carefully from each tray and washed thoroughly with running tap water. Data was recorded for each treatment at 14 days after sowing (DAS) on different parameters. Vigour Index (VI) was computed using the following formula of Baki and Anderson [30]:

Vigor index $=($ Mean shoot length + Mean root length $) \times \%$ Germination

\section{Laboratory Experiments (Blotter Method)}

The harvested seeds of each year were tested by blotter method for seed health test to detect seed borne pathogens associated with seed of each sample [31]. Each seed borne infection was recorded and expressed in percentage [32].

\section{Statistical Analysis}

The recorded data on different parameters were analyzed by using MSTAT-C computer program to find out the significance of variation resulting from experimental treatments. The difference between the treatment means were evaluated for significance using Duncan's Multiple Range Test (DMRT) following the procedure of Gomez and Gomaz [33].

\section{Results}

\section{Analysis of Disease Severity and Incidence}

The highest reduction of 85.00 and $87.69 \%$ in severity of narrow brown leaf spot of rice was recorded at 110 DAT with foliar application of Potent 250 EC $(0.1 \%)$ followed by BAU-Biofungicide (2\%) (85.19 \& 81.54\%) in 2012 and 2013 years, respectively, while the lowest incidence was observed (5.92 \& 6.04\%) in Potent (0.1\%) followed by BAUBiofungicide (2\%) presented in Table 1 . The low disease severity $(5.00 \%)$ and incidence $(10.21 \%)$ of narrow brown leaf spot disease were also noted at 110 DAT in Bavistin $(0.1 \%)$ in 2013. Minimum disease severity and incidence in bacterial leaf blight were obtained as 3.00 and $5.83 \%$ at 110 DAT, respectively with BAU-Biofungicide (3\%) in 2012 followed by Potent $(0.1 \%$ and $0.05 \%)$. The good effect of Bavistin in controlling bacterial leaf blight disease was found in this experiment as shown in Table 2.

\begin{tabular}{|c|c|c|c|c|c|c|c|c|c|c|c|c|}
\hline \multirow{4}{*}{$\begin{array}{l}\text { Treatment } \\
\text { (dose) }\end{array}$} & \multicolumn{12}{|c|}{ Narrow Brown Leaf Spot } \\
\hline & \multicolumn{6}{|c|}{ Disease severity (\%) } & \multicolumn{6}{|c|}{ Disease incidence (\%) } \\
\hline & \multicolumn{2}{|c|}{ At 80 DAT } & \multicolumn{2}{|c|}{ At 95 DAT } & \multicolumn{2}{|c|}{ At 110 DAT } & \multicolumn{2}{|c|}{ At 80 DAT } & \multicolumn{2}{|c|}{ At 95 DAT } & \multicolumn{2}{|c|}{ At 110 DAT } \\
\hline & 2012 & 2013 & 2012 & 2013 & 2012 & 2013 & 2012 & 2013 & 2012 & 2013 & 2012 & 2013 \\
\hline \multirow{2}{*}{$\begin{array}{c}\text { BAU-Biofungicide } \\
(2 \%)\end{array}$} & $4.47 \mathrm{c}$ & $4.00 \mathrm{c}$ & $4.23 \mathrm{e}$ & $4.00 \mathrm{~d}$ & $2.27 \mathrm{~g}$ & $3.00 \mathrm{e}$ & $9.18 \mathrm{~d}$ & $9.82 \mathrm{~d}$ & $10.40 \mathrm{e}$ & $11.92 \mathrm{~d}$ & $6.23 \mathrm{~g}$ & 7.16ef \\
\hline & -40.4 & -50 & -63.44 & -66.67 & -85.19 & -81.54 & -44.02 & -47.6 & -62.35 & -60.87 & -82.85 & -82.41 \\
\hline \multirow{2}{*}{\begin{tabular}{|c|} 
BAU-Biofungicide \\
$(3 \%)$ \\
\end{tabular}} & $4.53 \mathrm{c}$ & \multirow[b]{2}{*}{ - } & $4.13 \mathrm{e}$ & \multirow[b]{2}{*}{ - } & $2.37 \mathrm{~g}$ & & $9.13 \mathrm{~d}$ & \multirow{2}{*}{ - } & $10.39 \mathrm{e}$ & - & $6.30 \mathrm{~g}$ & \\
\hline & -39.6 & & -64.3 & & -84.54 & & -44.33 & & -62.38 & & -82.86 & \\
\hline \multirow{2}{*}{ Garlic (1\%) } & $5.27 \mathrm{bc}$ & $5.50 \mathrm{bc}$ & $6.97 \mathrm{~cd}$ & $7.50 \mathrm{c}$ & $8.67 \mathrm{~cd}$ & $9.00 \mathrm{c}$ & $13.04 \mathrm{bc}$ & $13.51 \mathrm{bc}$ & $16.55 \mathrm{c}$ & $18.69 \mathrm{c}$ & $23.27 \mathrm{c}$ & $23.37 \mathrm{c}$ \\
\hline & -29.73 & -31.25 & -39.76 & -37.5 & -43.44 & -44.62 & -20.49 & -27.91 & -40.08 & -38.64 & -35.95 & -42.59 \\
\hline \multirow{2}{*}{ Garlic (2\%) } & $5.00 \mathrm{bc}$ & & $6.00 \mathrm{~d}$ & & $7.22 \mathrm{de}$ & \multirow[b]{2}{*}{ - } & $11.86 \mathrm{c}$ & \multirow{2}{*}{-} & $13.65 \mathrm{~d}$ & - & $18.08 \mathrm{~d}$ & \\
\hline & -33.33 & & -48.14 & & -52.90 & & -27.68 & & -50.58 & & -35.95 & \\
\hline \multirow{2}{*}{ Neem (1\%) } & $6.07 \mathrm{~b}$ & 6.50ab & $9.00 \mathrm{~b}$ & $9.75 \mathrm{~b}$ & $11.38 \mathrm{~b}$ & $11.75 \mathrm{~b}$ & $14.15 \mathrm{~b}$ & $15.80 \mathrm{~b}$ & $22.12 b$ & $24.88 \mathrm{~b}$ & $28.00 \mathrm{~b}$ & $30.35 b$ \\
\hline & -19.07 & -18.75 & -22.21 & -18.75 & -25.77 & -28.8 & -13.72 & -15.69 & -19.91 & -18.32 & -22.93 & -25.45 \\
\hline \multirow{2}{*}{ Neem (2\%) } & $6.00 \mathrm{~b}$ & & $8.20 \mathrm{bc}$ & & $10.12 b c$ & & $13.53 \mathrm{bc}$ & & $19.88 \mathrm{~b}$ & - & $24.32 \mathrm{c}$ & \\
\hline & -20 & & -29.13 & & -33.99 & & -17.5 & & -28.02 & & -33.06 & \\
\hline \multirow{2}{*}{$\begin{array}{c}\text { Bavistin DF } \\
(0.1 \%)\end{array}$} & $5.10 \mathrm{bc}$ & $5.25 \mathrm{bc}$ & $6.07 \mathrm{~d}$ & $6.25 \mathrm{~cd}$ & $5.02 \mathrm{f}$ & $5.00 \mathrm{~d}$ & $11.81 \mathrm{c}$ & $11.15 \mathrm{~cd}$ & $13.16 \mathrm{~d}$ & $14.70 \mathrm{~d}$ & $10.26 \mathrm{f}$ & $10.21 \mathrm{e}$ \\
\hline & -32 & -34.38 & -47.54 & -47.92 & -67.25 & -69.23 & -27.99 & -40.5 & -52.35 & -51.74 & -71.76 & -74.92 \\
\hline \multirow{2}{*}{$\begin{array}{c}\text { Bavistin DF } \\
(0.05 \%)\end{array}$} & $5.67 \mathrm{bc}$ & $6.00 \mathrm{~b}$ & $7.00 \mathrm{~cd}$ & $7.50 \mathrm{c}$ & $6.26 \mathrm{ef}$ & $6.50 \mathrm{~d}$ & $12.53 \mathrm{bc}$ & $13.31 \mathrm{bc}$ & $16.17 \mathrm{c}$ & $18.86 \mathrm{c}$ & $13.24 \mathrm{e}$ & $14.80 \mathrm{~d}$ \\
\hline & -24.4 & -25 & -39.5 & -37.5 & -59.17 & -60 & -23.6 & -28.98 & -41.46 & -38.08 & -63.56 & -63.65 \\
\hline
\end{tabular}




\begin{tabular}{|c|c|c|c|c|c|c|c|c|c|c|c|c|}
\hline $\begin{array}{c}\text { Potent 250 EC } \\
(0.1 \%)\end{array}$ & $4.47 \mathrm{c}$ & $4.00 \mathrm{c}$ & $4.10 \mathrm{e}$ & $4.00 \mathrm{~d}$ & $2.30 \mathrm{~g}$ & $2.00 \mathrm{e}$ & $9.18 \mathrm{~d}$ & $10.23 \mathrm{~d}$ & $10.29 \mathrm{e}$ & $11.80 \mathrm{~d}$ & $5.92 \mathrm{~g}$ & $6.04 \mathrm{df}$ \\
\cline { 2 - 13 } & -40.4 & -50 & -64.56 & -66.67 & -85 & -87.69 & -44.02 & -45.41 & -62.74 & -61.26 & -83.7 & -85.16 \\
\hline $\begin{array}{c}\text { Potent 250 EC } \\
(0.05 \%)\end{array}$ & $4.50 \mathrm{c}$ & $4.00 \mathrm{c}$ & $4.00 \mathrm{e}$ & $4.50 \mathrm{~d}$ & $2.40 \mathrm{~g}$ & $2.50 \mathrm{e}$ & $9.14 \mathrm{~d}$ & $10.49 \mathrm{~d}$ & $10.39 \mathrm{e}$ & $12.49 \mathrm{~d}$ & $5.97 \mathrm{~g}$ & $7.28 \mathrm{ef}$ \\
\cline { 2 - 12 } & -40 & -50 & -65.43 & -62.5 & -84.34 & -84.62 & -44.27 & -44.02 & -62.38 & -59 & -83.57 & -82.12 \\
\hline Control (water) & $7.50 \mathrm{a}$ & $8.00 \mathrm{a}$ & $11.57 \mathrm{a}$ & $12.00 \mathrm{a}$ & $15.33 \mathrm{a}$ & $16.25 \mathrm{a}$ & $16.40 \mathrm{a}$ & $18.74 \mathrm{a}$ & $27.62 \mathrm{a}$ & $30.46 \mathrm{a}$ & $36.33 \mathrm{a}$ & $40.71 \mathrm{a}$ \\
\hline
\end{tabular}

Table 1: Effect of BAU-Biofungicide, extracts of garlic and neem, Bavistin and Potent on severity and incidence of narrow brown leaf spot disease of rice cv BRRI dhan29 in 2012 and 2013.

In a column, figures having same letter(s) do not differ significantly at $5 \%$ level of significance by Duncan's multiple range tests. DAT $=$ Days after Transplanting

Data represent the means of three replications

Data in parentheses indicate $\%$ disease severity and \% disease incidence reduction over control

$(-)=$ Not tested in 2013

\begin{tabular}{|c|c|c|c|c|c|c|c|c|c|c|c|c|}
\hline \multirow{3}{*}{ Treatment (dose) } & \multicolumn{6}{|c|}{ Disease severity (\%) } & \multicolumn{6}{|c|}{ Disease incidence (\%) } \\
\hline & \multicolumn{2}{|c|}{ At 80 DAT } & \multicolumn{2}{|c|}{ At 95 DAT } & \multicolumn{2}{|c|}{ At 110 DAT } & \multicolumn{2}{|c|}{ At 80 DAT } & \multicolumn{2}{|c|}{ At 95 DAT } & \multicolumn{2}{|c|}{ At 110 DAT } \\
\hline & 2012 & 2013 & 2012 & 2013 & 2012 & 2013 & 2012 & 2013 & 2012 & 2013 & 2012 & 2013 \\
\hline \multirow{2}{*}{\begin{tabular}{|c|} 
BAU-Biofungicide \\
$(2 \%)$ \\
\end{tabular}} & $4.00 \mathrm{~b}$ & $4.0 \mathrm{c}$ & $5.00 \mathrm{de}$ & $5.00 \mathrm{e}$ & $3.33 \mathrm{e}$ & $3.50 \mathrm{e}$ & $9.17 \mathrm{c}$ & $7.75 \mathrm{c}$ & $8.02 \mathrm{c}$ & $7.00 \mathrm{~d}$ & $6.08 \mathrm{~g}$ & $5.50 \mathrm{e}$ \\
\hline & -27.27 & -38.46 & -34.81 & -42.86 & -67.76 & -67.44 & -19.06 & -32.61 & -45.55 & -56.03 & -64.36 & -69.44 \\
\hline \multirow{2}{*}{\begin{tabular}{|c|}
$\begin{array}{c}\text { BAU-Biofungicide } \\
(3 \%)\end{array}$ \\
\end{tabular}} & $4.00 \mathrm{~b}$ & \multirow{2}{*}{ - } & $4.67 \mathrm{e}$ & \multirow{2}{*}{ - } & $3.00 \mathrm{e}$ & & $9.00 \mathrm{c}$ & \multirow{2}{*}{ - } & $7.99 \mathrm{c}$ & \multirow{2}{*}{ - } & $5.83 \mathrm{~g}$ & \multirow{2}{*}{ - } \\
\hline & -27.27 & & -39.11 & & -70.96 & & -20.56 & & -45.76 & & -65.83 & \\
\hline \multirow{2}{*}{ Garlic (1\%) } & $4.50 \mathrm{ab}$ & $5.50 \mathrm{ab}$ & $7.00 \mathrm{ab}$ & $7.25 \mathrm{~b}$ & $6.87 \mathrm{~b}$ & $7.50 \mathrm{~b}$ & $10.33 \mathrm{~b}$ & $10.50 \mathrm{ab}$ & $11.87 \mathrm{ab}$ & $12.17 \mathrm{~b}$ & $13.20 \mathrm{~b}$ & $13.63 b$ \\
\hline & -18.18 & -15.38 & -8.74 & -17.14 & -33.49 & -30.23 & -8.83 & -8.7 & -19.42 & -23.56 & -22.63 & -24.28 \\
\hline \multirow{2}{*}{ Garlic (2\%) } & $4.00 \mathrm{~b}$ & \multirow{2}{*}{ - } & $6.67 \mathrm{abc}$ & \multirow{2}{*}{-} & $6.33 \mathrm{bc}$ & & $9.83 \mathrm{bc}$ & \multirow{2}{*}{-} & $10.50 \mathrm{bc}$ & \multirow{2}{*}{ - } & $11.33 \mathrm{c}$ & \multirow{2}{*}{ - } \\
\hline & -27.27 & & -13.04 & & -38.72 & & -13.24 & & -28.72 & & -33.59 & \\
\hline \multirow{2}{*}{ Neem (1\%) } & $4.50 \mathrm{~b}$ & $5.0 \mathrm{bc}$ & $6.67 \mathrm{abc}$ & $7.00 \mathrm{bc}$ & $6.33 \mathrm{bc}$ & $7.25 b c$ & $10.33 \mathrm{~b}$ & $10.25 \mathrm{ab}$ & $10.39 \mathrm{bc}$ & $9.48 c$ & $10.00 \mathrm{~d}$ & $10.00 \mathrm{c}$ \\
\hline & -18.18 & -23.08 & -13.04 & -20 & -38.72 & -32.56 & -8.83 & -10.87 & -29.46 & -40.45 & -41.38 & -44.44 \\
\hline \multirow{2}{*}{ Neem (2\%) } & $4.25 \mathrm{~b}$ & \multirow{2}{*}{ - } & 6.33abcd & \multirow{2}{*}{-} & $6.00 \mathrm{bcd}$ & & $9.50 \mathrm{bc}$ & \multirow{2}{*}{-} & $10.08 \mathrm{bc}$ & - & 9.36de & - \\
\hline & -22.73 & & -17.47 & & -41.92 & & -16.15 & & -31.57 & - & -45.13 & - \\
\hline Bavistin DF ( & $5.00 \mathrm{ab}$ & $4.50 \mathrm{bc}$ & 5.67bcde & 6.00cde & $5.33 \mathrm{bcd}$ & $5.75 \mathrm{~cd}$ & $9.40 \mathrm{bc}$ & $10.00 \mathrm{ab}$ & $10.27 \mathrm{bc}$ & $9.50 \mathrm{c}$ & $8.58 \mathrm{ef}$ & $8.00 \mathrm{~cd}$ \\
\hline Davisem Dit $(0.1 / 0)$ & -9.09 & -30.77 & -26.08 & -31.43 & -48.4 & -46.51 & -17.04 & -13.04 & -30.28 & -40.33 & -49.71 & -55.56 \\
\hline & $5.00 \mathrm{ab}$ & $5.0 \mathrm{bc}$ & 6.00bcde & $6.25 \mathrm{bcd}$ & $5.67 \mathrm{bcd}$ & $6.00 \mathrm{bcd}$ & $10.00 \mathrm{bc}$ & $10.50 \mathrm{ab}$ & $10.50 \mathrm{bc}$ & $10.16 \mathrm{c}$ & 9.33de & $8.85 \mathrm{~cd}$ \\
\hline Bavls & -9.09 & -23.08 & -21.77 & -28.57 & -45.11 & -44.17 & -11.74 & -8.7 & -37.02 & -36.18 & -45.31 & -50.83 \\
\hline Potent $250 \mathrm{EC}$ & $4.00 \mathrm{~b}$ & $4.25 c$ & 5.67bcde & 5.50de & 4.33de & $5.25 \mathrm{~d}$ & $9.33 \mathrm{bc}$ & $9.25 b c$ & $9.83 \mathrm{bc}$ & $8.75 \mathrm{c}$ & $7.61 \mathrm{f}$ & 7.25de \\
\hline$(0.1 \%)$ & -27.27 & -34.62 & -26.08 & -37.14 & -58.08 & -51.16 & -17.65 & -19.57 & -33.27 & -45.04 & -55.39 & -59.72 \\
\hline Potent 250 EC & $4.50 \mathrm{ab}$ & $4.5 \mathrm{bc}$ & $5.33 \mathrm{cde}$ & 5.75de & $4.67 \mathrm{cde}$ & $5.50 \mathrm{~d}$ & $9.83 \mathrm{bc}$ & $9.50 \mathrm{abc}$ & $10.49 \mathrm{bc}$ & $9.25 \mathrm{c}$ & $7.87 \mathrm{f}$ & $7.50 \mathrm{cde}$ \\
\hline$(0.05 \%)$ & -18.18 & -30.77 & -30.51 & -34.29 & -57.79 & -48.84 & -13.24 & -17.39 & -28.78 & -41.9 & -53.87 & -58.33 \\
\hline Control (water) & $5.50 \mathrm{a}$ & $6.50 \mathrm{a}$ & $7.67 a$ & $8.75 a$ & $10.33 a$ & $10.75 a$ & $11.33 a$ & $11.50 \mathrm{a}$ & $14.73 a$ & $15.92 \mathrm{a}$ & $17.06 \mathrm{a}$ & $18.0 \mathrm{a}$ \\
\hline
\end{tabular}

Table 2: Effect of different treatments on severity and incidence of Bacterial Leaf Blight disease of rice cv BRRI dhan29 in 2012 and 2013

In a column, figures having same letter(s) do not differ significantly at 5\% level of significance by Duncan's multiple range tests. DAT $=$ Days after Transplanting

Data represent the means of three replications

Data in parentheses indicate $\%$ disease severity and \% disease incidence reduction over control

$(-)=$ Not tested in 2013 


\section{Evaluation of Seed Quality Improvement}

BAU-Biofungicide $(2 \%)$ and Potent $(0.1 \%)$ resulted in maximum (96.33\%) germination. Higher increase (30.85\%) in normal seedlings was found with BAU-Biofungicide (3\%) in 2012 as well as in Potent $250 \mathrm{EC}(0.1 \%)$ having increase of $32.83 \%$ over control in 2013. Highest reduction of diseased seedling (54.55\%) over control was achieved both in BAU-
Biofungicide (2\%) and Potent $(0.1 \%)$. Potent $(0.1 \%)$ also exhibited the highest shoot weight $(40.67 \mathrm{mg})$ and root weight $(37.33 \mathrm{mg})$, while BAU-Biofungicide $(2 \%)$ showed shoot weight $40.00 \mathrm{mg}$ and root weight $36.00 \mathrm{mg}$ in 2012 and 2013, respectively. Highest increase in vigor index (37.49\%) was appeared with BAU-Biofungicide (3\%) followed by Potent 250 EC (0.1\%) compared to control (Table 3).

\begin{tabular}{|c|c|c|c|c|c|c|c|c|c|c|c|c|}
\hline \multirow{3}{*}{$\begin{array}{c}\text { Treatment } \\
\text { (dose) }\end{array}$} & \multirow{2}{*}{\multicolumn{2}{|c|}{ Germination (\%) }} & \multirow{2}{*}{\multicolumn{2}{|c|}{$\begin{array}{c}\text { Normal } \\
\text { seedling (\%) }\end{array}$}} & \multirow{2}{*}{\multicolumn{2}{|c|}{$\begin{array}{c}\text { Diseased } \\
\text { seedling (\%) }\end{array}$}} & \multirow{2}{*}{\multicolumn{2}{|c|}{$\begin{array}{l}\text { Shoot weight } \\
\text { (mg) }\end{array}$}} & \multicolumn{2}{|c|}{ Root weight } & \multirow{2}{*}{\multicolumn{2}{|c|}{ Vigour index }} \\
\hline & & & & & & & & & & & & \\
\hline & 2012 & 2013 & 2012 & 2013 & 2012 & 2013 & 2012 & 2013 & 2012 & 2013 & 2012 & 2013 \\
\hline \multirow{2}{*}{\begin{tabular}{|c|} 
BAU- \\
Biofungicide \\
$(2 \%)$ \\
\end{tabular}} & $96.33 a$ & $96.00 \mathrm{ab}$ & 86.67ab & $86.00 \mathrm{a}$ & $5.00 \mathrm{c}$ & $5.00 \mathrm{~d}$ & $40.00 \mathrm{abc}$ & $39.67 \mathrm{a}$ & $35.00 \mathrm{a}$ & 36.00ab & $2125.00 \mathrm{ab}$ & $2136.62 a$ \\
\hline & $(+14.68)$ & $(+12.94)$ & $(+29.36)$ & $(+30.30)$ & $(-50.00)$ & $(-54.55)$ & $(+21.21)$ & $(+23.97)$ & $(+29.63)$ & $(+36.73)$ & $(+35.87)$ & $+34.92)$ \\
\hline \multirow{2}{*}{\begin{tabular}{|c|} 
BAU- \\
Biofungicide \\
$(3 \%)$ \\
\end{tabular}} & $96.00 \mathrm{a}$ & & $87.67 a$ & & $4.67 c$ & - & $39.67 \mathrm{abc}$ & & $35.00 \mathrm{a}$ & & $2150.40 \mathrm{a}$ & \\
\hline & $(+14.29)$ & & $(+30.85)$ & & $(-53.30)$ & & $(+20.21)$ & & $(+29.63)$ & & $(+37.49)$ & \\
\hline \multirow{2}{*}{ Garlic (1\%) } & 89.33ab & $90.00 \mathrm{abc}$ & $73.66 c$ & $75.00 \mathrm{bc}$ & $9.67 \mathrm{a}$ & $8.67 \mathrm{~b}$ & $36.00 \mathrm{bcd}$ & $36.00 \mathrm{ab}$ & $29.00 \mathrm{bcd}$ & 28.67de & $1736.67 \mathrm{de}$ & $1766.75 \mathrm{bc}$ \\
\hline & $(+6.35)$ & $(+5.88)$ & $(+9.94)$ & $(+13.64)$ & $(-3.30)$ & $(-21.18)$ & $(+9.09)$ & $(+12.50)$ & $(+7.41)$ & $(+8.89)$ & $(+11.04)$ & $(+11.57)$ \\
\hline \multirow{2}{*}{ Garlic (2\%) } & $90.00 \mathrm{ab}$ & & $76.67 \mathrm{c}$ & & $8.00 \mathrm{~b}$ & - & 36.33abcd & & $31.00 \mathrm{bc}$ & & 1830.92cde & \\
\hline & $(+7.14)$ & & $(+14.43)$ & & $(-20.00)$ & & $(+10.09)$ & & $(+14.81)$ & & $(+17.07)$ & \\
\hline \multirow{2}{*}{ Neem $(1 \%)$} & 86.33ab & $86.00 \mathrm{bc}$ & $70.00 \mathrm{c}$ & $69.00 \mathrm{bc}$ & $9.33 \mathrm{a}$ & $9.00 \mathrm{~b}$ & $35.00 \mathrm{~d}$ & $34.33 \mathrm{bc}$ & $28.67 \mathrm{~cd}$ & 29.00de & $1671.67 \mathrm{de}$ & $1660.46 \mathrm{bc}$ \\
\hline & $(+2.77)$ & $(+1.18)$ & $(+4.48)$ & $(+4.55)$ & $(-6.70)$ & $(-18.18)$ & $(+6.06)$ & $+7.28)$ & $(+6.19)$ & $(+10.14)$ & $(+6.88)$ & $(+4.85)$ \\
\hline \multirow{2}{*}{ Neem (2\%) } & 87.33ab & & $73.33 c$ & & $8.00 \mathrm{~b}$ & - & $35.67 \mathrm{~cd}$ & & $30.00 \mathrm{bcd}$ & & $1747.90 \mathrm{de}$ & \\
\hline & $(+3.96)$ & & $(+9.45)$ & - & $(-20.00)$ & & $(+8.09)$ & & $(+11.11)$ & & $(+11.76)$ & \\
\hline \multirow{2}{*}{$\begin{array}{c}\text { Bavistin DF } \\
(0.1 \%)\end{array}$} & $90.00 \mathrm{ab}$ & $89.67 \mathrm{abc}$ & $77.33 \mathrm{bc}$ & $77.00 \mathrm{~b}$ & $7.67 \mathrm{~b}$ & $7.33 c$ & 37.00abcd & 36.33ab & $32.67 \mathrm{ab}$ & $33.00 \mathrm{bc}$ & 1920.58abcd & 1899.50ab \\
\hline & $(+7.14)$ & $(+5.49)$ & $(+15.42)$ & $(+16.67)$ & $(-23.30)$ & $(-33.36)$ & $(+12.12)$ & $(+13.53)$ & $(+21.00)$ & $(+25.33)$ & $(+22.80)$ & $(+19.95)$ \\
\hline \multirow{2}{*}{\begin{tabular}{|c} 
Bavistin DF \\
$(0.05 \%)$ \\
\end{tabular}} & 88.67ab & 87.67abc & $74.67 \mathrm{bc}$ & $74.00 \mathrm{bc}$ & $7.67 \mathrm{~b}$ & $8.00 \mathrm{bc}$ & $36.00 \mathrm{bcd}$ & $35.00 \mathrm{bc}$ & $30.00 \mathrm{bcd}$ & 30.33cd & 1807.33cde & $1774.77 \mathrm{bc}$ \\
\hline & $(+5.56)$ & $(+3.14)$ & $(+11.45)$ & $(+12.12)$ & $(-23.30)$ & $(-27.27)$ & $(+9.09)$ & $(+9.38)$ & $(+11.11)$ & $(+15.19)$ & $(+15.56)$ & $(+12.07)$ \\
\hline \multirow{2}{*}{$\begin{array}{l}\text { Potent } 250 \\
\text { EC }(0.1 \%)\end{array}$} & $96.33 a$ & $96.33 a$ & $88.66 a$ & $87.67 \mathrm{a}$ & $5.00 \mathrm{c}$ & $5.00 \mathrm{~d}$ & $40.67 a$ & $40.00 \mathrm{a}$ & $36.00 \mathrm{a}$ & 37.33a & $2127.32 \mathrm{ab}$ & $2125.93 a$ \\
\hline & $(0.1 \%)$ & $(+13.33)$ & $(+32.33)$ & $(+32.83)$ & $(-50.00)$ & $(-54.55)$ & $(+23.24)$ & $(+25.00)$ & $(+33.33)$ & $(+41.78)$ & $(+36.02)$ & $(+34.25)$ \\
\hline \multirow{2}{*}{$\begin{array}{l}\text { Potent } 250 \\
\text { EC }(0.05 \%)\end{array}$} & $0.00 \mathrm{a}$ & $96.33 a$ & a & 86.33a & $5.33 \mathrm{c}$ & $6.00 \mathrm{~d}$ & $40.50 \mathrm{ab}$ & $39.33 a$ & $35.67 \mathrm{a}$ & $37.00 \mathrm{a}$ & $2103.60 \mathrm{abc}$ & $2100.97 a$ \\
\hline & $(+14.29)$ & $(+13.33)$ & $(+30.85)$ & $(+30.80)$ & $(-46.70)$ & $(-45.45)$ & $(+22.73)$ & $(+22.91)$ & $(+32.11)$ & $(+40.52)$ & $(+34.50)$ & $(+32.67)$ \\
\hline $\begin{array}{l}\text { Control } \\
\text { (water) }\end{array}$ & $84.00 \mathrm{~b}$ & $85.00 \mathrm{c}$ & $67.00 \mathrm{c}$ & $66.00 \mathrm{c}$ & $10.00 \mathrm{a}$ & $11.00 \mathrm{a}$ & $33.00 \mathrm{~d}$ & $32.00 \mathrm{c}$ & $27.00 \mathrm{~d}$ & $26.33 \mathrm{e}$ & $1564.00 \mathrm{~d}$ & $1583.60 \mathrm{c}$ \\
\hline
\end{tabular}

Table 3: Effect of different treatments on germination(\%) and vigour index at 14 days after sowing of harvested seeds of rice cv. BRRI dhan29 following tray method during Boro season in 2012 and 2013.

In a column, figures having same letter(s) do not differ significantly at 5\% level of significance by DMRT

Data represent the means of three replications

Data in parentheses indicate \% increased (+) and \% decreased (-) over control

DAS = Days after sowing

$(-)=$ Not tested in 2013 


\section{Seed Borne Pathogen Determination}

The harvested seeds of different treatments were found to be detected as seed borne fungi, viz. B. oryzae, Curvularia lunata, Fusarium oxysporum, Fusarium moniliforme, Sarocladium oryzae and Penicillium sp. (Table 4). The lowest $(2.00 \%)$ seed borne infection of B. oryzae was observed in harvested seeds as foliar application of BAU-Biofungicide (3\%) followed by BAU-Biofungicide $(2.00 \%)$ and Potent 250 EC $(0.1 \%)$. The highest $(65.22 \%)$ reduction of seed borne infection of $C$. lunata was determined with BAU-
Biofungicide (2\%) over control followed by Neem (2\%). Minimum infection $(10.50 \%)$ of $F$. oxysporum was marked in BAU-Biofungicide $(3 \%)$ followed by BAU-Biofungicide $(2 \%)$ and Potent $(0.1 \& 0.05 \%)$. Maximum reduction of $F$. moniliforme $(72.22 \%)$ was recorded with BAU-Biofungicide (3\%) followed by BAU-Biofungicide (2\%) over control. Seed borne infection of $S$. oryzae was not identified in Potent $(0.1$ and $0.05 \%$ ). Hundred percent reduction of Penicillium $s p$. was found with BAU-Biofungicide (2\%) followed by BAUBiofungicide (3\%) and Potent (0.1 \& $0.05 \%$ ) over control.

\begin{tabular}{|c|c|c|c|c|c|c|c|c|c|c|c|c|}
\hline \multirow{2}{*}{$\begin{array}{c}\text { Treatment } \\
\text { (dose) }\end{array}$} & \multicolumn{2}{|c|}{ Bipolaris oryzae } & \multicolumn{2}{|c|}{$\begin{array}{c}\text { Curvularia } \\
\text { Iunata }\end{array}$} & \multicolumn{2}{|c|}{$\begin{array}{c}\text { Fusarium } \\
\text { oxysporum }\end{array}$} & \multicolumn{2}{|c|}{$\begin{array}{c}\text { Fusarium } \\
\text { moniliforme }\end{array}$} & \multicolumn{2}{|c|}{$\begin{array}{c}\text { Sarocladium } \\
\text { oryzae }\end{array}$} & \multicolumn{2}{|c|}{ Penicillium sp. } \\
\hline & 2012 & 2013 & 2012 & 2013 & 2012 & 2013 & 2012 & 2013 & 2012 & 2013 & 2012 & 2013 \\
\hline \multirow{2}{*}{$\begin{array}{c}\text { BAU-Biofungicide } \\
(2 \%)\end{array}$} & $2.50 \mathrm{e}$ & $2.00 \mathrm{e}$ & $4.75 \mathrm{e}$ & $4.00 \mathrm{e}$ & $12.75 \mathrm{e}$ & $10.00 \mathrm{~d}$ & $1.50 \mathrm{~d}$ & $2.50 \mathrm{~d}$ & $1.50 \mathrm{~cd}$ & $1.00 \mathrm{~d}$ & $0.00 \mathrm{e}$ & $1.50 \mathrm{e}$ \\
\hline & $(-70.59)$ & $(-66.67)$ & $(-64.81)$ & $-65.22)$ & $(-32.89)$ & $(-42.03)$ & $(-66.67)$ & $(-50.0)$ & $(-57.14)$ & $(-73.33)$ & $(-100.00)$ & $(-75.00)$ \\
\hline \multirow{2}{*}{$\begin{array}{c}\text { BAU-Biofungicide } \\
(3 \%)\end{array}$} & $2.00 \mathrm{e}$ & - & $5.00 \mathrm{e}$ & \multirow{2}{*}{-} & $10.50 \mathrm{f}$ & \multirow{2}{*}{-} & $1.25 \mathrm{~d}$ & \multirow{2}{*}{-} & $1.00 \mathrm{~d}$ & \multirow{2}{*}{-} & $1.00 \mathrm{~d}$ & \multirow{2}{*}{-} \\
\hline & $(-76.47)$ & & $(-62.96)$ & & $(-44.74)$ & & $(-72.22)$ & & $(-71.43)$ & & $(-80.95)$ & \\
\hline \multirow{2}{*}{ Garlic (1\%) } & $6.50 \mathrm{~b}$ & $4.25 \mathrm{bc}$ & $7.00 \mathrm{~d}$ & $6.50 \mathrm{~cd}$ & $16.00 \mathrm{bc}$ & $12.50 \mathrm{c}$ & $2.50 \mathrm{c}$ & $2.75 \mathrm{~cd}$ & $2.00 \mathrm{bc}$ & $2.00 \mathrm{c}$ & $1.00 \mathrm{~d}$ & 2.25cde \\
\hline & $(-23.53)$ & $(-29.17)$ & $(-48.15)$ & $(-43.48)$ & $(-15.79)$ & $(-27.54)$ & $(-44.44)$ & $(-45.0)$ & $(-42.86)$ & $(-46.67)$ & $(-80.95)$ & $(-62.50)$ \\
\hline \multirow{2}{*}{ Garlic (2\%) } & $5.50 \mathrm{c}$ & - & $5.50 \mathrm{e}$ & \multirow[b]{2}{*}{ 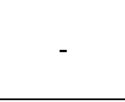 } & $16.25 \mathrm{bc}$ & \multirow{2}{*}{ - } & $2.50 \mathrm{c}$ & \multirow{2}{*}{-} & $1.00 \mathrm{~d}$ & \multirow{2}{*}{-} & $0.00 \mathrm{e}$ & \multirow{2}{*}{ - } \\
\hline & $(-35.29)$ & & $(-59.26)$ & & $(-14.47)$ & & $(-44.44)$ & & $(-71.43)$ & & $(-100.00)$ & \\
\hline \multirow{2}{*}{ Neem (1\%) } & $4.00 \mathrm{~d}$ & $3.50 \mathrm{~cd}$ & $5.25 \mathrm{e}$ & $5.00 \mathrm{de}$ & $16.50 \mathrm{bc}$ & $15.00 \mathrm{~b}$ & $2.75 b c$ & $3.50 \mathrm{bc}$ & $2.00 \mathrm{bc}$ & $3.00 \mathrm{~b}$ & $2.50 \mathrm{~b}$ & $3.50 \mathrm{~b}$ \\
\hline & $(-52.94)$ & $(-41.67)$ & $(-61.11)$ & $(-56.52)$ & $(-13.16)$ & $(-13.04)$ & $(-38.89)$ & $(-30.0)$ & $(-42.86)$ & $(-20.00)$ & $(-52.38)$ & $(-41.67)$ \\
\hline \multirow{2}{*}{ Neem (2\%) } & $4.00 \mathrm{~d}$ & - & $5.00 \mathrm{e}$ & \multirow{2}{*}{ - } & $14.00 \mathrm{de}$ & \multirow{2}{*}{-} & $3.00 \mathrm{bc}$ & \multirow{2}{*}{-} & $2.00 \mathrm{bc}$ & \multirow{2}{*}{-} & $2.00 \mathrm{bc}$ & \multirow{2}{*}{ - } \\
\hline & $(-52.94)$ & & $(-62.96)$ & & $(-26.32)$ & & $(-33.33)$ & & $(-42.86)$ & & $(-61.90)$ & \\
\hline \multirow{2}{*}{ Bavistin DF (0.1\%) } & $8.50 \mathrm{a}$ & $4.75 b$ & $11.00 \mathrm{c}$ & $8.00 \mathrm{bc}$ & $17.75 \mathrm{ab}$ & $17.00 \mathrm{a}$ & $3.50 \mathrm{~b}$ & $3.75 b$ & $2.50 \mathrm{~b}$ & $2.00 \mathrm{c}$ & $2.50 \mathrm{~b}$ & $3.00 \mathrm{bc}$ \\
\hline & $(-0.00)$ & $(-20.83)$ & $(-18.52)$ & $(-30.43)$ & $(-6.58)$ & $(-1.45)$ & $(-22.22)$ & $(-25.0)$ & $(-28.57)$ & $(-46.67)$ & $(-52.38)$ & $(-50.00)$ \\
\hline \multirow{2}{*}{$\begin{array}{c}\text { Bavistin DF } \\
(0.05 \%)\end{array}$} & $8.50 \mathrm{a}$ & $5.00 \mathrm{ab}$ & $11.50 \mathrm{bc}$ & $8.50 \mathrm{~b}$ & $18.50 \mathrm{a}$ & $16.50 \mathrm{ab}$ & $4.50 \mathrm{a}$ & $5.00 \mathrm{a}$ & $3.50 \mathrm{a}$ & $3.00 \mathrm{~b}$ & $2.00 \mathrm{bc}$ & $3.50 \mathrm{~b}$ \\
\hline & $(-0.00)$ & $(-16.67)$ & $(-14.81)$ & $(-26.09)$ & $(-2.63)$ & $(-4.35)$ & $(-0.00)$ & $(-0.00)$ & $(-0.00)$ & $(-20.00)$ & $(-61.90)$ & $(-41.67)$ \\
\hline \multirow{2}{*}{$\begin{array}{c}\text { Potent } 250 \text { EC } \\
(0.1 \%) \\
\end{array}$} & $3.50 \mathrm{~d}$ & $2.25 \mathrm{e}$ & $12.50 \mathrm{ab}$ & $8.25 b$ & $13.25 \mathrm{de}$ & $11.00 \mathrm{~cd}$ & $2.50 \mathrm{c}$ & $3.00 \mathrm{bcd}$ & $0.00 \mathrm{e}$ & $0.00 \mathrm{e}$ & $1.50 \mathrm{~cd}$ & $2.00 \mathrm{de}$ \\
\hline & $(-58.82)$ & $(-62.50)$ & $(-7.41)$ & $(-28.26)$ & $(-30.26)$ & $(-36.23)$ & $(-44.44)$ & $(-40.0)$ & $(-100.00)$ & $(-100.00)$ & $(-71.43)$ & $(-66.67)$ \\
\hline Potent 250 EC & $5.00 \mathrm{c}$ & 3.00de & $13.50 \mathrm{a}$ & $9.00 \mathrm{~b}$ & $14.75 \mathrm{~cd}$ & $11.75 \mathrm{c}$ & $3.00 \mathrm{bc}$ & $3.50 \mathrm{bc}$ & $0.00 \mathrm{e}$ & $0.00 \mathrm{e}$ & $1.00 \mathrm{~d}$ & $2.50 \mathrm{~cd}$ \\
\hline$(0.05 \%)$ & $(-41.18)$ & $(-50.00)$ & $(-0.00)$ & $(-21.74)$ & $(-22.37)$ & $(-31.88)$ & $(-33.33)$ & $(-30.0)$ & $(-100.0)$ & $(-100.00)$ & $(-80.95)$ & $(-58.33)$ \\
\hline Control (water) & $8.50 \mathrm{a}$ & $6.00 \mathrm{a}$ & $13.50 \mathrm{a}$ & $11.50 \mathrm{a}$ & $19.00 \mathrm{a}$ & $17.25 \mathrm{a}$ & $4.50 \mathrm{a}$ & $5.00 \mathrm{a}$ & $3.50 \mathrm{a}$ & $3.75 a$ & $5.25 a$ & $6.00 \mathrm{a}$ \\
\hline
\end{tabular}

Table 4: Effect of different treatments on germination (\%) and seed borne fungi in seeds of cv BRRI dhan29 following Blotter method during Boro season in 2012 and 2013.

In a column, figures having same letter(s) do not differ significantly at $5 \%$ level of significance by DMRT

Data represent the means of four replications

Data in parentheses indicate \% increased (+) and \% decreased (-) over control

$(-)=$ Not tested in 2013

\section{Discussion}

Mahmud and Hossain [18] reported that BAUBiofungicide (2\%) and Tilt 250 EC $(0.1 \%)$ resulted in significant reduction of disease incidence of narrow brown leaf spot disease. This finding was complementing with Zhou and Uppala [34] who observed the lowest severity of narrow brown leaf spot in propiconazole under field conditions. Mukherjee and Maheswari [35] reported that Trichoderma harzianum was found to have highly antagonistic effect on 
narrow brown leaf spot of paddy. These findings were also supported by Razu and Hossain [15]. Mahmud et al. [13] reported that BAU-Biofungicide (2\%) exhibited the lowest disease severity of bacterial leaf blight disease when it was applied as foliar spray in the field. Similar findings were also supported by Tang, et al. [36], Gangwar \& Sinha, and Razu \& Hossain $[37,15]$. Significant reduction of disease severity in bacterial leaf blight of rice was noted with the application of Trichoderma harzianum due to higher phenolic content production on rice leaves as reported by Gangwar and Sinha [37]. Similar observation was also reported by Kumawat, et al. [38].

Mahmud and Hossain [18] reported that application of BAU-Biofungicide (2\%) and Potent 250 EC $(0.1 \%)$ signified maximum germination and highest seedling of harvested seeds. These findings were in accordance with the observation of Biswas, et al. [39] who reported that Trichoderma treated rice seeds showed maximum germination (92\%) and increased shoot and root length. This result was similar to the findings of Brotman, et al. [40] and López-Bucio, et al. [41]. They observed that Trichoderma spp. induced the plant to release phytoalexins and phenols that provided tolerance to abiotic stresses and enhanced the development of root system. Mahmud and Hossain [18] also reported that BAU-Biofungicide $(2 \%)$ remarked maximum reduction of diseased seedling, and highest increase (36.17\%)) in vigour index of harvested seeds of rice cv BR11. These findings were also reported by Hossain, et al. (2015).

Mahmud and Hossain [18] tested the efficacy of $T$. harzianum against $B$. oryzae and observed the significant growth reduction of rice brown spot pathogen. Similar findings were also reported by Sarkar, et al. [42], Biswas, et al. [39] and Biswas, et al. [43]. Biswas et al. [43] also observed $70 \%$ disease reduction when the seeds were treated with Trichoderma spp. Mahmud and Hossain [25] reported that BAU-Biofungicide (2\%) reduced (67.57\%) seed borne infection of $C$. lunata of harvested seeds when it was applied as foliar spray. This finding was also supported by Jat and Agalave [44]. Gwa and Nwankiti [45] reported that $T$. harzianum significantly inhibited the growth of seed borne pathogen (F. oxysporum). F. oxysporum was reduced significantly with propiconazole $(0.1 \%)$ as reported by Manasa, et al. [46]. Trichoderma species showed the maximum retardation in growth $(52.54 \%)$ of seed borne infection of $F$. moniliforme which was studied by Gwa and Nwankiti [47], and they also observed that T. harzianum was found to be antagonistic on F. moniliforme due to antibiosis and mycoparasitism. Bora and Ali (2019) reported that significant growth inhibition (65.21\%) of T. harzianum against $S$. oryzae was observed. The highest reduction in seed borne infection of $S$. oryzae was also found in propiconazole $(0.1 \%)$ as the reporting of Mahmud and Hossain [18].
Trichoderma showed antagonistic effect against Penicillium spp. as reported by Jat and Agalave [44]. Similar findings were reported by Borrás and Aguilar [48].

BAU-Biofungicide (2\%) was highly effective in reducing disease incidence of narrow brown leaf spot and bacterial leaf blight in the field. BAU-Biofungicide and Potent $(0.1 \%)$ also increased germination of seeds, seedling growth and vigor index, and inhibited seed borne pathogens of harvested seeds of BAU-Biofungicide and Potent $(0.1 \%)$ sprayed plot.

\section{Acknowledgment}

I would gratefully acknowledge the funding authority, Director of National Agricultural Technology Project, Department of Agricultuaral Extension, Dhaka, Bangladesh.

\section{References}

1. FAO (2018).

2. Gangopadhyay (1983) Current concepts on fungal disease of rice, pp: 205-208

3. Sah DN, Rush MC (1988) Physiologic races of Cercospora oryzae in the Southern United States. Plant Disease 72(3): 262-264.

4. Firoz MJ (2001) Induction of resistance to rice against some major diseases. M.S. thesis, Dept. Plant Pathology, Bangladesh Agricultural University, Mymensingh, Bangladesh, pp: 50.

5. Wamishe Y, Cartwright R, Lee F (2017) Management of Rice Diseases. Arkansas rice production handbook, Chapter 11, pp: 141.

6. Groth D (2013) Why Cercospora, blast and bacterial panicle blight are more severe some years. Rice disease newsletter. Louisiana State University Agricultural Center, Baton Rouge.

7. Elings A, Reddy PR, Marimuthu T, Rossing WAH, Jansen MJW, Teng PS(1997) Rice bacterial leaf blight: field experiments, systems analysis and damage coefficients. Field Crops Research 51(1-2): 113-131.

8. Mew TW, Alvarez AM, Leach JE, Swings J (1993) Focus on bacterial blight of rice. Plant Disease 77(1): 5-12.

9. FAO (2002) FAO statistic India.

10. Rajarajeswari NVL, Muralidharan K (2006) Assessments of farm yield and district production loss from bacterial leaf blight epidemics in rice. Crop Protection 25(3): 244252. 
11. Mia MAT, Nahar MA (2000) Status of rice seed health in Bangladesh and farmers' seed production and management scenario. In: Mew TW, Cotton B, (Eds.), Seed Health and Seed associated Microorganisms for Rice Disease Management, International Rice Research Institute, Los Banos, Laguna, Philippines, pp: 81-86.

12. Fakir GA, Mia MAT (2004) The quality of Farmer saved rice seeds in Bangladesh. National workshop on seed health improvement. BARC, Dhaka.

13. Mahmud H, Hossain I, Ahmad MU (2016) Efficacy of BAU-Biofungicide on brown spot and bacterial leaf blight disease and vigour index of rice. Bangladesh Journal of plant pathology 32(1-2): 25-31.

14. Mew TW, Leung Hei, Savary S, Casiana MVC, Leach Jan E (2004) Looking ahead in rice disease research and management. Critical reviews and plant Sciences 23(2): 103-127.

15. Razu MAU, Hossain I (2015) Eco-friendly management of rice diseases. International Journal of Applied Science and Biotechnology 3(1): 80-88.

16. Palaniyandi V, Immanuel JE, Gnanamanickam SS, Thomashow L (2006) Biological control of rice bacterial blight by plant associated bacteria producing 2,4diacetylphloroglucinol. Canadian Journal of Microbiology 52(1): 56-65.

17. Gangwar GP, Sinha AP (2012) Evaluation of Trichoderma spp. and fluorescent pseudomonads for the management of bacterial leaf blight of rice. Indian Phytopathology 65(1): 89-91.

18. Mahmud H, Hossain I (2017) Comparative efficacy of BAU-Biofungicide and synthetic fungicides in management of diseases of rice (Oryza sativa L.) for quality seed production. Brazilian Journal of Botany 40(2): 389-397.

19. Karlsson M, Atanasova L, Jensen DF, Zeilinger S (2017) Necrotrophic mycoparasites and their genomes. Microbiology Spectrum 5(2): FUNK-0016-2016.

20. Hjeljord L, Tronsmo A (1998) Trichoderma and Gliocladium in bio-logical control: an overview. In: Harman GE, Kubicek CP, (Eds.), Taylor and Francis, London, pp: 131-152.

21. Harman GE, Howell CR, Viterbo A, Chet I, Lorito M (2004) Trichoderma species-opportunistic, avirulent plant symbionts. Nature Review Microbiology 2(1): 43-56.

22. Irkin R, Korukluoglu M (2007) Control of Aspergillus niger with garlic, onion and leek extracts. Afr. J.
Biotechnol 6(4): 384-387.

23. Reddy KRN, Nurdijati SB, Salleh B (2010) An overview of plant-derived products on control of mycotoxigenic fungi and mycotoxins. Asian Journal of Plant Science 9(3): 126-133.

24. Hossain MM, Hossain I, Khalequzzaman KM (2015) Effect of seed treatment with Biological Control Agent against Bipolaris leaf blight of wheat. International Journal of Scientific Research in Agricultural Sciences 2: 151-158.

25. Mahmud H, Hossain I (2016) Effects of plant extracts, BAU-Biofungicide and fungicides on quality and health of seed. Bangladesh Journal of Botany 45(3): 677-684.

26. Hossain I (2011) BAU-Biofungicide: Unique Eco-friendly Means and New Dimension of Plant Disease Control in Bangladesh: Leaflet published from the Dept. of Plant Pathology, Bangladesh Agricultural University, Mymensingh, pp: 8-11.

27. BRRI (2004) Modern Rice Cultivation. Bangladesh Rice Research Institute Gazipur, pp: 26-27.

28. IRRI (1996) Standard Evaluation System of Rice, $4^{\text {th }}$ (Edn.), International Rice Research Institute, P.O. Box 933, 1099 Manila, Philippines, pp: 52.

29. Dasgupta MK (1988) Principles of Plant Pathology. Applied Publisher Pvt. Ltd., New Delhi, pp: 706.

30. Abdul-Baki AA, Anderson JD (1973) Vigour determination of soybean seed by multiple criteria. Crop Science 13(6): 630-633.

31. ISTA (1996) International rules for Seed Testing. Seed Science and Technology 4: 3-49.

32. Agarwal PC, Mortensen CM, Mathur SB (1989) Seedborne diseases and seed health testing of rice. Technical Bulletin No. 3, Phytopathlogical Paper No. 30, CAB International Mycological Institute (CMI) Kew, Surrey, UK, pp: 58-59.

33. Gomez KA, Gomez AA (1984) Statistical procedures for agricultural research, $2^{\text {nd }}(E d n$.), Wiley, New York.

34. Zhou XZ, Uppala SS (2015) Field efficacy of new fungicides for management of narrow brown leaf spot of rice. Conference proceeding of the Biol Disease ManageMycology, Pasadena, California, USA.

35. Mukherjee B, Maheswari NU (2018) Biological control of narrow brown Leaf spot and leaf smut disease in paddy crops by some antagonistic fungi. Global Journal of Medical Research 18(2): 1-12. 
36. Tang J, Tian B, Ling Xia M, Ping W, Hong C (2001) Biocontrol of Rhizoctonia solani with Trichoderma spp. Chinese Rice Res Newsletter 9: 8.

37. Gangwar GP, Sinha AP (2014) Effect of fungal and bacterial bioagent application on total phenolic content in rice leaves pre-inoculated with Xanthomonas oryzae pv. oryzae (Uyeda and Ishiyama) Dowson. Journal of Applied and Natural Science 6(1): 254-257.

38. Kumawat GL, Biswas SK, Srivastava, SSL (2012) Biochemical evidence of defense response in paddy induced by bio-agents against brown leaf spot pathogen. Indian Phytopathology 61(2): 197-203.

39. Biswas SK, Ratan V, Srivastava SSL, Ramesh S (2008) Influence of seed treatment with biocides and foliar spray with fungicides for management of Brown leaf spot and Sheath blight of paddy. Indian Phytopathology 61(1): 55-59.

40. Brotman Y, Landau U, Cuadros-Inostroza A, Takayuki T, Fernie AR, et al. (2013) Trichoderma plant root colonization: escaping early plant defense responses and activation of the antioxidant machinery for saline stress tolerance. PLoS Pathog 9(4): 10.

41. López-Bucio J, Pelagio-Flores R, Herrera-Estrella A (2015) Trichoderma as biostimulant: exploiting the multilevel properties of a plant beneficial fungus. Science and Horticulture196: 109-123.

42. Sarkar D, Mandal R, Roy P, Taradar J, Dasgupta B (2014) Management of brown spot disease of rice by using safer fungicides and some Bioagents. The Bioscan 9(1): 437441.

43. Biswas C, Srivastava SSL, Biswas SK (2010) Effectof biotic, abiotic and botanical inducers on crop growth and severity of brown spot in rice. Indian Phytopathology 63(2): 187-191.

44. Jat JG, Agalave HR (2013) Antagonistic properties of Trichoderma species against oilseed-borne fungi. Science Research Reporter 3(2): 171-174.

45. Gwa VI, Nwankiti AO (2018) Evaluation of antagonistic effect of Trichoderma Harzianum against Fusarium oxysporum causal agent of white yam (Dioscorearotundata poir) tuber rot. Trends in Technical \& Scientific Research 1(1): 12-18.

46. Manasa BG, Somashekara YM, Shankara K, Swamy C (2017) Efficacy of Fungicides in Control of Fusarium oxysporum f. sp. dianthi, the Cause of wilt in carnation. International Journal of Current Microbiology and Applied Sciences 6(10): 2559-2565.

47. Gwa VI, Nwankiti AO (2017) In Vitro Antagonistic Potential of Trichoderma harzianum for Biological Control of Fusarium moniliforme isolated from Dioscorea rotundata Tubers. Virology \& Mycology 6(2): 1-8.

48. Borrás AD, Aguilar RV (1990) Biological control of Penicillium digitatum by Trichoderma viride on postharvest citrus fruits. International journal of food microbiology 11(2): 179-183. 